\title{
Étude de l'interaction enseignant - élèves en physique au lycée. Enseigner comme agir sur les performances didactiques des élèves
}

Studying teacher - pupils interaction in physics at the high school. Teaching as acting on the pupils didactic performances

\section{Abdelkarim Zaid}

\section{OpenEdition \\ Journals}

Édition électronique

URL : http://journals.openedition.org/educationdidactique/1627

DOI : 10.4000/educationdidactique.1627

ISSN : 2111-4838

\section{Éditeur}

Presses universitaires de Rennes

\section{Édition imprimée}

Date de publication : 30 novembre 2012

Pagination : 125-146

ISBN : 978-2-7535-2254-1

ISSN : $1956-3485$

\section{Référence électronique}

Abdelkarim Zaid, «Étude de l'interaction enseignant - élèves en physique au lycée. Enseigner comme agir sur les performances didactiques des élèves », Éducation et didactique [En ligne], 6-3 | novembre 2012, mis en ligne le 30 novembre 2014, consulté le 08 décembre 2020. URL : http:// journals.openedition.org/educationdidactique/1627 ; DOI : https://doi.org/10.4000/ educationdidactique.1627 


\title{
ÉTUDE DE L'INTERACTION ENSEIGNANT - ÉLÈVES EN PHYSIQUE AU LYCÉE. ENSEIGNER COMME AGIR SUR LES PERFORMANCES DIDACTIQUES DES ÉLÈVES
}

\author{
Abdelkarim Zaid (CIREL, IUFM Nord Pas de Calais-Université d'Artois)
}

\begin{abstract}
Résumé: L’unité d'enregistrement vidéo issue de la base de données VISA analysée ici montre une séance d'enseignement en physique sous de fortes contraintes de situation. Le contenu en jeu relève du thème intitulé « l'Univers » en classe de seconde et porte essentiellement sur des modèles pour interpréter et caractériser la lumière émise par des étoiles. Cette étude focalise l'interaction enseignant - élèves en concevant l'action de l'enseignant comme action sur les performances didactiques des élèves. Elle met en évidence que l'action de l'enseignant prend en compte les performances des élèves et manifeste les caractéristiques d'un processus de modélisation, c'est-à-dire une dynamique entre référent empirique et modèle construit. Par ailleurs, les performances manifestées par les élèves mettent en évidence l'appropriation de la signification de certains modèles et leur adaptation à de différentes situations, mais elles ne permettent pas de conclure quant à une construction intellectuelle modélisante.
\end{abstract}

Mots clés: Vidéo ; interaction ; sciences physiques ; modélisation ; performances didactiques.

Abdelkarim Zaid

\section{Introduction}

Les discours institutionnels et de recherche portant sur l'enseignement des sciences à l'école insistent sur les modalités d'enseignement qui privilégient l'investigation, le travail en groupe, le débat, la confrontation des idées, la formulation d'hypothèses, etc. Ces discours contrastent fortement avec les contraintes effectives et quotidiennes liées aux pratiques d'enseignement: les exigences matérielles et les modalités pédagogiques qu'implique l'enseignement des sciences, la planification temporelle de la progression des apprentissages, la programmation des séances dans la journée comme dans l'année, le nombre d'élèves et leur disposition en salle de classe, la disponibilité des salles spécialisées, la préparation de l'enseignant, etc.

De ce point de vue, l'enseignement des sciences se déroule couramment dans des situations fortement contraintes (Crahay, 1989), telle que la situation d'enseignement analysée dans ce texte. En effet, l'unité d'enregistrement vidéo issue de la base de données VISA, qui est à la base de cette analyse, fait apparaître une situation d'enseignement où les élèves en classe entière sont disposés en rangées parallèles au bureau de l'enseignant et où l'enseignant, souvent devant ses élèves, corrige des activités «d'application» en sciences physiques, sur le thème de «l'Univers». Et ce qui nous a frappé en visionnant la vidéo de manière itérative, et en prenant en compte le discours de l'enseignant commentant sa propre pratique $^{1}$, c'est ce complexe de contraintes ${ }^{2}$ qui a pesé sur le travail de l'enseignant et sur ses interactions avec les élèves tout au long de la séance. Ce constat nous a conduit à poser les questions suivantes: comment l'action de l'enseignant se construit-elle et évolue-t-elle au sein de ce complexe de contraintes? Quel lien y a-t-il entre progressivité du contenu et ce que l'enseignant observe et interprète du faire de ses élèves? Qu'apprennent les élèves dans une telle situation?

D'autres motivations ont suscité ces questions. En particulier le fait que, souvent, l'enseignement dans ce type de situation est considéré comme «frontal», « transmissif», « classique » ou encore "non constructiviste», donc ne permettant pas des apprentissages "valables», notamment en sciences. Ce genre d'enseignement est donc rapidement disqualifié et demeure non pensé. Or, les situations contraintes comme celle analysée ici sont une réalité quotidienne des enseignants d'aujourd'hui et ce type de situations ne constitue pas un cas particulier ou «en voie d'extinction ${ }^{3} »$. Du coup, il nous semble intéressant d'explorer ce qui 
se joue dans ce genre de situations, et de le faire en caractérisant les modes d'interaction entre l'enseignant et les élèves d'une part et les modes de gestion des élèves et de leurs apprentissages d'autre part, dans une visée compréhensive.

Bien entendu, le processus d'enseignement et d'apprentissage ne tourne pas à vide, il porte sur un contenu spécifique, ici l'analyse spectrale, qui relève du thème intitulé «l'Univers» en classe de seconde. Ce contenu implique un enseignement qui fait appel à des activités sur des modèles; à l'utilisation et la manipulation de dispositifs expérimentaux (prisme, CD, spectroscope, etc.) ; à la manipulation de supports de représentation (schémas, photos, maquettes, etc.); à l'observation et donc à des interactions entres les élèves et l'enseignant visant à la formulation d'hypothèses. Il s'agit donc ici d'explorer comment cet enseignement est actualisé dans la séance observée. En ce sens, nous tenterons de décrire comment l'enseignant fait progresser le «système didactique» (Johsua et Dupin, 1993; Daunay, 2007) en s'appuyant sur ce qu'il observe et interprète du faire des élèves au sein d'un complexe de contraintes. Le faire des élèves étant manifesté par des gestes, des paroles, des silences, et étant interprété par les acteurs de la situation, notamment l'enseignant, comme des réussites, des échecs, des apprentissages ou au moins comme une participation (De Marcellus, 2011).

\section{Cadre théorique}

En vue de décrire la construction de l'action de l'enseignant pour faire progresser sa séance, nous nous appuierons sur un modèle didactique de l'action d'enseignement, un modèle qui rend compte de l'interdépendance de l'action de l'enseignant et de celle des élèves. En effet, au sein du système d'interactions élève, enseignant, contenu, contexte matériel et social, nous nous intéressons en particulier à l'évolution de l'action didactique de l'enseignant telle qu'elle est suscitée par différentes actions des élèves: des gestes, des paroles, des productions écrites, etc., mais aussi des contraintes liées à la préparation de l'enseignant ou à la situation de classe. Ces gestes, paroles, silences et les différentes productions des élèves sont analysées ici en tant que performances didactiques (Daunay, 2008 ; Lahanier-Reuter, 2008; Reuter, 2011).

Dans un premier temps, nous clarifierons l'interdépendance entre action de l'enseignant et performances didactiques des élèves en concevant l'action d'enseignement comme une double médiation (Lenoir, 1996). Dans un second temps, nous expliciterons le sens et l'usage de la notion de performance didactique.

\section{La pratique de l'enseignant comme double médiation}

Le contenu en jeu dans une situation d'enseignement/apprentissage est marqué, déterminé par les rapports sociaux caractéristiques de la situation de sa production. En outre, dans une perspective qui évacue toute appropriation immédiate, le rapport de l'élève à ce contenu est un rapport d'objectivation (Freitag, 1986, Lenoir, ibid.). Ce rapport se réalise à travers une double médiation: d'une part, une médiation cognitive interne au processus d'objectivation élève-contenu; d'autre part une médiation externe, la médiation didactique (Lenoir, ibid.). Un processus d'objectivation s'établit ainsi entre l'élève et le contenu et requiert la mise en œuvre de processus médiateurs (Maubant et Zaid, à paraître) dont l'action didactique de l'enseignant. Celle-ci agit donc en tant que médiation didactique sur le processus d'objectivation élève-contenu dans lequel l'écriture, la parole ou la manipulation des objets détiennent un rôle essentiel. L'action de l'élève, n'est plus une simple forme de perturbation du cours «normal» de l'action de l'enseignant, elle en est un élément déterminant. De ce fait, prendre en compte l'interdépendance de ces deux actions ne se limite pas à prendre en compte la seule marge de variations induite par l'activité des élèves (Bautier, 2006).

L'action d'enseignement en tant que double médiation implique donc que l'enseignant agit sur le faire de l'élève, et plus spécifiquement sur la manifestation du rapport d'objectivation qui s'établit entre l'élève et le contenu (Lenoir, 2009). Si bien que l'avancement dans l'enseignement et dans l'apprentissage peut être conçu comme une succession ${ }^{4}$ de doubles médiations ${ }^{5}$. L'expression manifeste, interprétée par un acteur de la situation d'enseignement/apprentissage, du rapport de l'élève 
au contenu en tant que forme d'engagement dans la situation de classe est spécifiquement ce que nous considérons ici comme performance didactique. L'action de l'enseignant consiste ainsi à sélectionner parmi les performances didactiques des élèves celles qui lui permettent le mieux d'effectuer les cadrages nécessaires (Astolfi, 1992) et donc lui permettent de progresser dans le déploiement de son dispositif didactique et pédagogique. Cette manière de concevoir l'action de l'enseignant rejoint les idées d'hétérogénisation didactique (Sarrazay, 2008) et de visibilité didactique (Chopin, 2008) développées dans ce même numéro par M.P. Chopin.

\section{La performance didactique des élèves}

La construction de la notion de performance didactique s'appuie sur les acquis d'un séminaire de recherche ${ }^{6}$ qui a interrogé les notions de performances et apprentissages disciplinaires selon différents points de vue didactiques (Daunay, 2007.; Reuter, 2011). Elle sera mobilisée ici avec une visée heuristique. Au moins deux arguments peuvent être avancés pour justifier la discussion puis les propositions de reconstruction didactique de la notion de performance. D'une part, il y a le positionnement scientifico-social des didactiques en ce sens que celles-ci sont des disciplines de recherche «impliquées », appelées à prendre en charge des demandes sociales par rapport à l'évaluation des performances des élèves. Il faut souligner que des évaluations internationales centrées sur les performances des élèves, telles que PISA et TIMMS ${ }^{7}$, prennent de plus en plus d'importance sans que cette notion soit explicitée. D'autre part, l'analyse des performances des élèves traite d'un problème éminemment didactique, celui $\mathrm{du}$ fonctionnement des disciplines en ce qu'il formate l'interaction entre le faire des élèves et le faire des enseignants (Reuter, 2011).

La notion de performance est polysémique et son usage, en didactique comme dans d'autres champs de recherche, confond le faire et son résultat, la construction descriptive de ce qui est soumis à analyse et la compréhension, l'interprétation et l'évaluation de cette description (Reuter, ibid.). Cette confusion va de concert avec la naturalisation de cette notion très utilisée dans les tests d'évaluations nationaux et internationaux. La notion de performance didactique des élèves est mobilisée ici en tant qu'effet du système didactique (Reuter, ibid.) construit par un observateur (enseignant, chercheur, etc.) pour inférer les apprentissages des élèves. C'est un construit qui vise à rendre compte de la construction par l'élève du contenu d'enseignement (qui peut être des savoirs, des savoir-faire, des rapports à, des attitudes, des conduites et des compétences ou des rapports plus complexes tel que le rapport entre compétences, dynamique de l'élève et pratiques d'enseignement [Reuter, ibid.; Daunay, ibid.]). Il rend possible l'étude de ce qui, dans le faire des sujets didactiques, intéresse le chercheur et lui impose de passer par une perspective évaluative qui porte sur les contenus en jeu ou les mouvements au sein du système didactique (Daunay, ibid.). Dès lors, on peut considérer qu'une performance didactique offrirait une image des apprentissages de l'élève qui sont à l'origine de l'évolution et des transformations du système didactique.

Ces transformations sont à repérer à travers des indicateurs d'apprentissage manifestes, tels que les paroles, les gestes, l'usage d'outils, spécifiés par le contenu, mais aussi les silences ${ }^{8}$, en ce qu'ils peuvent constituer des événements qui font évoluer le système didactique. En tant qu'action ancrée dans la situation d'enseignement et d'apprentissage, la performance didactique est à caractériser d'abord en référence aux éléments déterminants de la situation, tels que les communications et les interactions entre les élèves et l'enseignant, la nature du curriculum et les activités scolaires qui en découlent ainsi que le dispositif pédagogique nécessaire pour les mettre en œuvre.

Pour conclure sur cette brève présentation de la notion de performance didactique, nous insistons sur son caractère heuristique, en particulier du fait que l'on considère le silence de l'élève à un moment donné d'une séance comme une performance didactique, malgré les questions épistémologiques et méthodologiques que cela pose et que nous n'évoquerons pas ici. En revanche, nous assumons ce choix en tant que critique d'une idéologie qui ne conçoit l'engagement scolaire que sur le mode de l'expression écrite ou orale, le silence n'étant pas considéré comme un faire «performatif».

\section{Performances didactiques et apprentissage des modèles et des processus de modélisation}


Comme nous le mentionnons précédemment, l'enjeu de la séance analysée ici est l'apprentissage d'un contenu qui comprend des savoirs, des démarches mais aussi des communications et des interactions sociales caractéristiques de tout processus de construction des savoirs en sciences physiques. D'une part, le programme de seconde organise ce contenu-en termes de notions comme les spectres d'émission et d'absorption, les raies d'émission ou d'absorption d'un atome ou d'un ion, etc. (M.E.N., 2010), soit principalement des modèles. D'autre part, le programme organise le contenu en termes de compétences telles que savoir qu'un corps chaud émet un rayonnement continu, savoir repérer une radiation caractéristique d'une entité chimique par sa longueur d'onde ou encore savoir utiliser un système dispersif pour visualiser des spectres d'émission et d'absorption, etc. (M.E.N., ibid.), soit principalement des compétences de modélisation. On voit bien l'importance que détiennent les modèles et les processus de modélisation dans ce contenu si bien que l'on peut considérer que l'enseignant viserait à ce que les performances didactiques, en tant que manifestation des apprentissages des élèves, possèdent les caractéristiques des processus de modélisation.

Martinand (2010) identifie trois caractéristiques essentielles des modèles (et donc des processus de modélisation): «Ils sont et restent hypothétiques; Ils sont modifiables pour s'adapter aux "besoins"; Ils sont pertinents pour certaines problématiques dans certains contextes» (Martinand, 2010, p. 20). Par ailleurs, dans une situation de modélisation, les tâches proposées aux élèves impliquent une dynamique de concordance des faits et des théories (Orange, 1997) ou des allers et retours entre ce que Martinand (ibid.) appelle référent empirique et modèle scientifique. L'enseignant est donc amené à préparer la tâche au regard de cette distinction et en ayant deux responsabilités didactiques. L'enseignant est amené, d'une part, à «effectuer les choix qui constituent le référent empirique pour les élaborations modélisantes: quels objets? Quels phénomènes? Quelles manipulations introduire? Quelles règles pour réussir les manipulations? Quels concepts, notions, concepts et représentations pour décrire les phénomènes? » (Martinand, ibid., p. 21). Il est amené d'autre part à expliciter les problèmes et les visées qui justifient la construction du modèle, préciser les outils symboliques utilisés, les théories qui servent de cadre, les règles d'utilisation et le champ de validité (Martinand, ibid.). L'action de l'enseignant sera donc analysée en la considérant comme action sur des performances didactiques des élèves, guidant leur activité entre référent empirique et modèle construit. Les performances didactiques des élèves, elles, seront analysées à la lumière des caractéristiques essentielles des processus de modélisation afin de préciser la nature et l'expression de ces processus.

\section{Méthodologie}

\section{Contexte de l'enregistrement vidéo}

Comme nous l'avons souligné précédemment, l'action de l'enseignant est d'abord contrainte par sa propre préparation qui prévoit un contenu théorique très dense au regard de la durée de la séance observée (54 minutes) avec la nécessité d'utiliser différents supports matériels et documentaires (spectre papier en noir et blanc et en couleur, vidéoprojecteur, tableau, etc.). La séance observée est programmée à la suite d'une séance de travaux pratiques en demi-classe qui, dans le cadre du thème «L'Univers» en classe de seconde, visait à une première initiation des élèves à l'analyse spectrale. À travers des manipulations et des observations, il s'est agi dans la première séance d'utiliser le spectroscope, d'étudier le spectre de la lumière émise par une ampoule à incandescence, d'interpréter la couleur des étoiles, d'observer et d'interpréter les spectres d'émission de différentes lampes et de déterminer la composition d'une étoile à l'aide de son spectre. La séance observée, elle, est consacrée à la correction d'activités d'application ${ }^{9}$ proposées lors de la séance de Travaux Pratiques. La correction de chaque application est introduite par des rappels rendus nécessaires par les questions des élèves, ou par des compléments de cours.

L'enregistrement vidéo de cette séance s'est effectué avec une ${ }^{10}$ caméra placée au fond de la salle de classe et focalisée sur l'enseignant. Par des mouvements de zoom, l'enregistrement se focalise tantôt sur l'enseignant, dont ce qu'il écrit au tableau, tantôt sur toute la classe. La possibilité de visionner l'enregistrement de manière itérative permet de saisir les interactions de l'enseignant avec les élèves de manière précise et dans leur ancrage temporel. C'est en ce sens que la vidéo est un instrument pertinent pour capter l'évolution 
du système didactique et, en particulier, pour rendre compte de l'interdépendance des actions de l'enseignant et des élèves. Bien entendu, l'évolution de l'action de l'enseignant ne consiste pas seulement en des évolutions spatiales ou temporelles, mais aussi en des évolutions thématiques en interaction avec (ou visant à) des performances didactiques spécifiques comme par exemple: comprendre un modèle de décomposition de la lumière, le mettre en œuvre pour classifier des étoiles au regard de leurs températures/couleurs ou pour les situer dans le plan $\mathrm{HR}^{11}$.

\section{Traitement des données}

La transcription et l'analyse de l'enregistrement vidéo ont été réalisées en utilisant le logiciel Transana ${ }^{12}$. La démarche d'analyse est conçue comme un dialogue entre d'une part, une construction théorique, un modèle interprétatif de l'action de l'enseignant tel que nous l'avons développé dans le cadre théorique; d'autre part, la pratique effective telle qu'elle est observée par le chercheur. Au cours de ce va-et-vient entre le visionnement de l'enregistrement vidéo et le cadre théorique, une grille a été construite en privilégiant le point de vue des performances didactiques des élèves. Les indicateurs de performances didactiques ne sont pas systématiquement interprétés comme des réussites ou des échecs et nous évitons donc de considérer la réussite comme indicateur exclusif d'une performance. Tout en s'appuyant sur les choix conceptuels relatés précédemment, la grille d'analyse prend aussi en compte l'observation directe de la séance d'enseignement. D'où la proposition de distinguer les performances didactiques en performances cognitives (à dominante de savoirs et de compétences notionnelles), performances techniques (à dominante d'usage d'objets ou de supports: cette catégorie n'a pas été renseignée car l'enregistrement vidéo analysé ne le permet pas), performances sociales (à dominante d'interactions avec les pairs ou avec l'enseignante) et ce que nous appellerons performances de silence (performances manifestées par un silence en situation d'écoute, d'attente suite à une question, de réponse à un rappel à l'ordre, etc.). Le tableau $n^{\circ} 1$ donne des exemples d'indicateurs de performances.

\begin{tabular}{|c|c|}
\hline $\begin{array}{l}\text { Performances } \\
\text { didactiques }\end{array}$ & Indicateurs \\
\hline $\begin{array}{l}\text { Les performances didac- } \\
\text { tiques cognitives }\end{array}$ & $\begin{array}{l}\text { - formuler, reformuler des énoncés de la consigne } \\
\text { - discuter en groupe des notions spécifiques au contenu en jeu } \\
\text { - manifester un savoir antérieur déjà construit } \\
\text { - commenter ou poser des questions par rapport à la consigne de la tâche à réaliser... etc. }\end{array}$ \\
\hline $\begin{array}{l}\text { Les performances didac- } \\
\text { tiques techniques }\end{array}$ & $\begin{array}{l}\text { - manipuler un matériel ou un support (prisme, CD, spectroscope, spectres sur papier, etc.). } \\
\text { - rendre compte, argumenter, justifier l'usage du spectre papier (collage sur cahier). }\end{array}$ \\
\hline $\begin{array}{l}\text { Les performances didac- } \\
\text { tiques de silence }\end{array}$ & $\begin{array}{l}\text { - Manifester un silence vis-à-vis d'une explication, d'une question ou d'un rappel à l'ordre de l'ensei- } \\
\text { gnant }{ }^{13} \text {. Manifester un silence exprimant un engagement dans la situation didactique. } \\
\text { - Manifester un silence vis-à-vis de l'explication ou d'une question d'un autre élève. }\end{array}$ \\
\hline $\begin{array}{l}\text { Les performances didac- } \\
\text { tiques sociales }{ }^{14}\end{array}$ & $\begin{array}{l}\text { - discuter avec l'enseignant ou avec les autres élèves de la consigne, de l'application à faire } \\
\text { - demander la parole pour poser ou répondre à une question liée à l'application à réaliser, par parole ou } \\
\text { par geste (lever le doigt). } \\
\text { - exprimer un accord/désaccord avec l'enseignant, avec un élève } \\
\text { - compléter, commenter une réponse de l'enseignant. }\end{array}$ \\
\hline
\end{tabular}

Tableau $n^{\circ} 1$ : indicateurs des différentes catégories de performances didactiques

La distinction de ces types de performances a structuré l'analyse de l'action de l'enseignant découpée en segments délimités par des événements remarquables (phases de la séance, types d'inter- vention, positions par rapport à l'élève ou dans la classe, etc.). La séance est découpée selon le synopsis suivant en trois phases qui ont marqué l'action de l'enseignante (tableau $\left.n^{\circ} 2\right)$ : 
ETUdE DE L'INTERACTION ENSEIGNANT - ÉLÈVES EN PHYSIQUE AU LYCÉE...

\section{Abdelkarim Zaid}

\begin{tabular}{|l|l|l|}
\hline Intitulé de la phase & \multicolumn{1}{|c|}{ Définition de la phase } & \multicolumn{1}{c|}{ Durée de la phase } \\
\hline Préparation & $\begin{array}{l}\text { préparation des élèves au début de la séance, énoncé et explication de } \\
\text { la consigne (ou la tâche) }\end{array}$ & $\begin{array}{l}\text { De 00 :00 :00 } \\
\text { à 0:09:25.3 }\end{array}$ \\
\hline Travail en groupe & réalisation de la tâche par les élèves en groupe (binômes) & $\begin{array}{l}\text { De 0:09:25.3 } \\
\text { à 0:23:44.3 }\end{array}$ \\
\hline Structuration & $\begin{array}{l}\text { Rappels, correction des 'activités d'application' et structuration des } \\
\text { savoirs }\end{array}$ & $\begin{array}{l}\text { De 0:23:44.3 } \\
\text { à 0:55:46.5 }\end{array}$ \\
\hline
\end{tabular}

Tableau $n^{\circ} 2$ : synopsis de la séance observée

Les actions de l'enseignant relatives à chaque phase sont ensuite découpées en segments délimités par des événements qui marquent des évolutions de l'action (la fin des rappels de cours, le début d'une consigne, des routines de rappels à l'ordre, etc.). Aux événements sont enfin associées les performances didactiques manifestées par les élèves, telles qu'ils les ont suscitées ou non, ce que montre le tableau suivant (tableau $\mathrm{n}^{\circ} 3$ ).

\begin{tabular}{|l|l|l|l|l|}
\hline \multicolumn{1}{|c|}{ Segment i } & \multicolumn{1}{|c|}{ Temps } & \multicolumn{1}{|c|}{$\begin{array}{c}\text { Événements délimitant le } \\
\text { segment i }\end{array}$} & $\begin{array}{l}\text { Performances manifestées par } \\
\text { les élèves }\end{array}$ & $\begin{array}{c}\text { Lien entre événements et } \\
\text { performances }\end{array}$ \\
\hline $\begin{array}{l}\text { Segment « Cr } \\
25 »\end{array}$ & $\begin{array}{l}\text { Début } \\
0: 10: 33.6\end{array}$ & $\begin{array}{l}\text { Événement (i-1:24): } \\
\text { L'enseignant s'arrête de cher- } \\
\text { cher sur son bureau les docu- } \\
\text { ments à distribuer et demande } \\
\text { aux élèves de travailler en } \\
\text { silence. }\end{array}$ & $\begin{array}{l}\text { Des discussions bruyantes } \\
\text { entre les élèves par rapport } \\
\text { aux documents qui manquent } \\
\text { (performance sociale) }\end{array}$ & $\begin{array}{l}\text { La modification de l'action } \\
\text { de l'enseignant (le rappel à } \\
\text { l'ordre) intervient lorsque les } \\
\text { discussions entre les élèves } \\
\text { deviennent bruyantes (perfor- } \\
\text { mance sociale). }\end{array}$ \\
\cline { 2 - 5 } & Fin: 0:10:59.2 & $\begin{array}{l}\text { Événement (i: 25): } \\
\text { L'enseignant finit rapidement } \\
\text { le rappel à l'ordre et quitte } \\
\text { son bureau pour répondre à la } \\
\text { question d'une élève qui pose } \\
\text { une question }\end{array}$ & $\begin{array}{l}\text { L'élève demande si l'activité } \\
\text { consiste à justifier pourquoi le } \\
\text { spectre est continu (performance } \\
\text { cognitive) }\end{array}$ & $\begin{array}{l}\text { La modification de l'action de } \\
\text { l'enseignant (se déplacer pour } \\
\text { répondre à l'élève) suite à la } \\
\text { question de l'élève (perfor- } \\
\text { mance cognitive). }\end{array}$ \\
\hline
\end{tabular}

Tableau $\mathrm{n}^{\circ} 3$ : Exemple de découpage et d'interprétation de l'action de l'enseignant « $\mathrm{Cr} »$.

Ces deux descriptions interdépendantes, celles des événements et des performances didactiques associées (ou non), nous ont permis de renseigner les conditions de l'évolution de l'action de l'enseignant et du système didactique en lien avec les performances didactiques des élèves. Le recours à la mesure fréquentielle s'appuie sur une hypothèse de corrélation entre l'occurrence d'un événement (action de l'enseignant) et un indicateur d'une performance didactique mobilisée par l'élève. Cependant, cette mesure n'a été utilisée que pour repérer les événements ou les performances à analyser de manière plus approfondie (Zaid, 2011).

\section{Résultats}

\section{Évolution de l'action de l'enseignant et performances didactiques des élèves}

Les performances didactiques des élèves ont été manifestées en termes de performances cognitives, sociales, et de silence. L'enregistrement vidéo ne permet pas de juger de la manifestation ou non des performances didactiques techniques. Le tableau ${ }^{\circ} 4$ fait apparaître que l'enseignant a dû composer avec des performances sociales, cognitives et de silence.

\begin{tabular}{|c|c|c|c|}
\hline Performances cognitives & Performances silences & Performances sociales & Total des occurrences \\
\hline$(36 \%)$ & $(24 \%)$ & $(40 \%)$ & $(100 \%)$ \\
\hline
\end{tabular}

Tableau $n^{\circ} 4$ : Total des occurrences des performances didactiques au cours de l'action de l'enseignant 
L'analyse des événements délimitant les segments de l'action de l'enseignant permet de repérer comment les performances didactiques ont été prises en compte ou non dans l'évolution de son action. Le tableau ${ }^{\circ} 5$ met en évidence que 125 événements parmi les 200 identifiés ont marqué des évolutions ou des modifications de l'action de l'enseignant suscitées par des performances didactiques des élèves.

\begin{tabular}{|rr|r|r|}
\hline $\begin{array}{l}\text { Événements liés à des prises de décisions } \\
\text { de l'enseignant }\end{array}$ & $\begin{array}{l}\text { Événements suscités par des perfor- } \\
\text { mances didactiques des élèves }\end{array}$ & Nombre total $^{15}$ d'événements \\
\hline 75 & 125 & $62 \%$ & 200 \\
\hline 38 & & $100 \%$ \\
\hline
\end{tabular}

Tableau $\mathbf{n}^{\circ} 5$ : Événements caractérisant l'évolution de l'action de l'enseignant

Ces événements ont consisté, par exemple, à s'interrompre (de parler par exemple) pour écouter une question ou un commentaire d'un élève, à changer de position (devant les élèves, à coté ou devant le tableau), à changer de support de travail (tableau noir, poster en couleur, document papier, vidéoprojecteur), à vérifier le travail d'un binôme qui annonce avoir terminé une question, etc. Ils ont consisté aussi en des événements qui portent sur le contenu: passer de l'explication des conditions de réalisation de la tâche à celle du contenu nécessaire à sa réalisation, du rappel des notions nécessaires à la réalisation de la tâche à l'introduction de nouvelles notions, etc.

Les 75 événements restants montrent une évolution de l'action de l'enseignant sans que celle-ci soit exclusivement suscitée par des performances didactiques des élèves. Il s'agit d'évolutions plutôt liées à des prises de décisions de l'enseignant, prenant en compte ou non des performances didactiques, prises de décisions déterminées par d'autres considérations professionnelles telle que la planification didactique, pédagogique, temporelle ou encore matérielle de la séance.

Durant les segments d'action de l'enseignant liés à des événements suscités par des performances des élèves, le tableau n ${ }^{\circ} 6$ fait apparaître qu'il y a presque autant de performances cognitives manifestées chez les élèves que de performances sociales (76 occurrences pour les premières et 71 pour les secondes). Mais il y a aussi beaucoup de moments de silence (36 occurrences).

\begin{tabular}{|c|c|c|c|}
\hline & Performances cognitives & Performances de silence & Performances sociales \\
\hline $\begin{array}{l}\text { Segments liés à des événements } \\
\text { suscités par des performances }\end{array}$ & 76 & $24 \%$ & $40 \%$ \\
\hline $\begin{array}{l}\text { Segments liés à des événements } \\
\text { suscités par des prises de décision }\end{array}$ & 21 & $24 \%$ & $40 \%$ \\
\hline
\end{tabular}

Tableau $n^{\circ} 6$ : Occurrences des Événements performances didactiques

Les Performances didactiques sociales ont consisté en des interactions entre les élèves ou avec l'enseignant, sans rapport direct avec le contenu: lever la main pour demander un document manquant; demander collectivement le document d'une activité particulière (l'activité 3); commenter la difficulté de l'activité (pas facile) et de la notion en jeu (spectre d'absorption); discuter avec l'enseignant la cause du manque de documents; demander de manière répétitive un document présentant le spectre en couleur.
Les Performances didactiques cognitives, elles, sont manifestées à travers des interactions qui visent à discuter en groupe des notions en jeu (poser une question par rapport à la signification des couleurs du spectre; à poser une question par rapport à une unité de mesure [distance entre les astres]) ; à manifester un savoir antérieur déjà construit (discuter par rapport au comportement de la rétine face au soleil; demander l'explication de la lumière des lucioles, expliquer le lien entre la lumière bleutée d'une étoile et donc sa grande température; à exprimer des 
commentaires ou des questions par rapport à la tâche à réaliser (commenter les explications de l'enseignant par rapport au spectre).

Aux segments d'actions de l'enseignant encadrés par ou liés à des événements suscités par des prises de décision, correspondent plus de performances sociales manifestées que de performances cognitives et de silence. Les performances cognitives se sont manifestées sous forme de questions par rapport à des points spécifiques du cours (questions par rapport aux raies noires dans le spectre de la lumière blanche). Par ailleurs, les performances sociales se sont manifestées sous forme de discussions collectives avec l'enseignant (nécessité d'un spectre en couleur pour la tâche à réaliser) ou de discussions « diffuses » et peu audibles entre les élèves.
Nous pouvons alors dire que l'évolution de l'action de l'enseignant, envisagée du point de vue des performances didactiques des élèves, est déterminée par les décisions prises par l'enseignant pour faire évoluer la séance à travers ses différentes phases (préparation, travail en groupe, structuration). En même temps, à l'intérieur de chaque phase, ce sont les performances didactiques des élèves qui constituent le moteur essentiel, mais non exclusif, de l'évolution du système didactique. L'action de l'enseignant conçue comme action sur les performances didactiques des élèves prend ainsi tout son sens. La figure $\mathrm{n}^{\circ} 4$ met en évidence la façon dont l'action de l'enseignant a évolué à l'intérieur de chaque activité, en lien avec les performances des élèves.

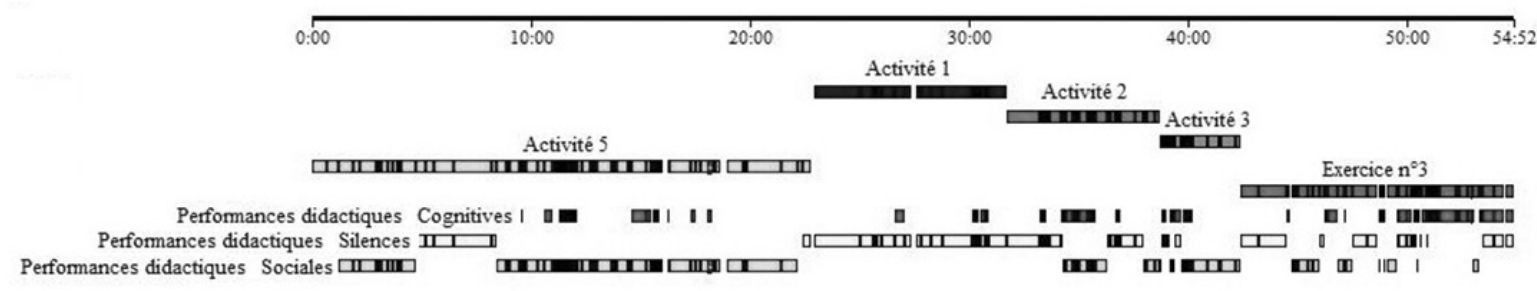

Figure $\mathrm{n}^{\circ} 4$ : Schéma de l'évolution temporelle de la séance: activités et performances

Les constats précédents nous permettent-ils de dire qu'au sein de la situation contrainte observée, l'enseignant dispose d'appuis possibles pour initier des interactions spécifiques à un enseignement portant sur les modèles et la modélisation? L'analyse de la séance qui nous a paru comme une situation d'enseignement «frontal » marquée par une omniprésence de l'enseignant et un statut passif des élèves a permis de dévoiler un enseignement dont l'évolution est plus subtile, prenant en compte les performances didactiques des élèves.

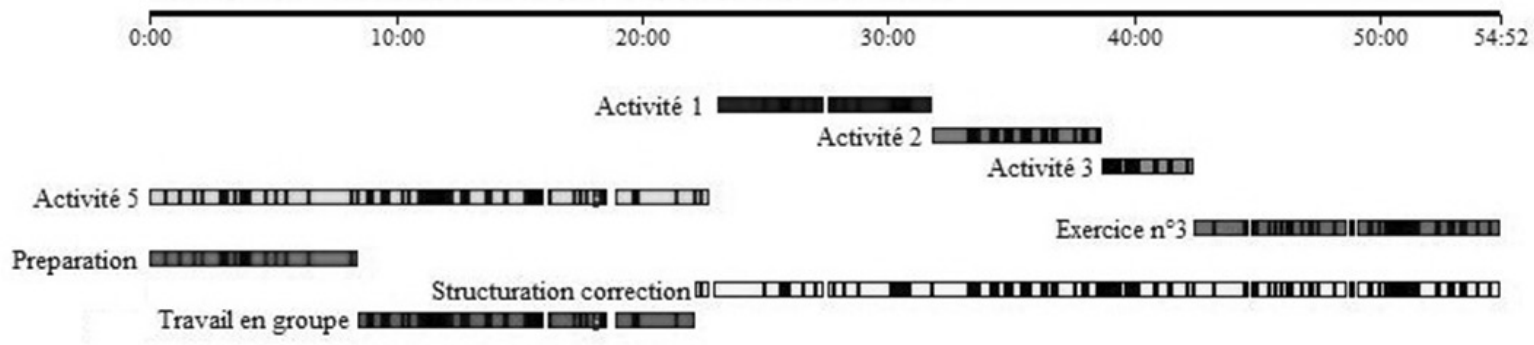

Figure $n^{\circ} 2$ : Schéma de l'évolution temporelle de la séance: phases et activités 


\section{Évolution de l'action de l'enseignant et processus de modélisation}

\section{Analyse globale}

Le graphe suivant met en évidence l'évolution de la séance à travers les trois phases identifiées. Nous caractérisons ici l'évolution de l'action didactique de l'enseignant lors de la correction des activités et exercices, à travers l'analyse des moments de présentation ou d'explication des consignes données aux élèves, qui ont balisé toutes leurs activités. Il ne s'agit pas d'une évolution linéaire: elle peut être analysée en trois niveaux, comme le montre en partie la figure $\mathrm{n}^{\circ} 2$.

Un premier niveau d'évolution correspond à l'avancement à travers les trois phases, dont les deux premières sont consacrées à l'énoncé et la distribution des compléments de ressources de l'activité 5. La troisième phase est caractérisée par un deuxième niveau d'évolution, cette fois à travers trois activités et un exercice. Un troisième niveau d'évolution se situe à l'intérieur de chaque activité et porte sur le contenu. Par exemple, l'enseignant passe des conditions de réalisation d'une activité aux notions nécessaires pour la réalisation:

« [Explication des conditions de réalisation, décision de l'enseignant] Alors c'est pas facile de les reconnaître, on les collera tout à l'heure. Ne les collez pas tout de suite, il y en aura peut-être en trop mais surtout ce qui vous paraît difficile [XXX] dans l'activité 5 à mon avis c'est que il faut prendre en compte à la fois le modèle sur les spectres d'émission et le modèle des spectres d'absorption [explication du contenu, décision de l'enseignant], parce que quand on analyse la lumière du soleil ou d'une étoile en général, on va avoir à prendre en compte à la fois l'émission mais aussi les zones noires en particulier. ».

Dans la même explication, l'enseignant change de support:

« [explication se référant à un document présentant plusieurs spectres de différentes lumières ] hein les radiations mono chromatiques c'est-à-dire les radiations de lumière qui correspondent à une seule couleur du spectre / de quoi est composée cette lumière quelles radiations et bien pour ça on utilise un dispositif dispersif un spectroscope et en gros on en a deux à notre disposition vous avez utilisé enfin vous avez surtout utilisé heu vous le prisme mais vous avez aussi utilisé le réseau parce que dans les petites boites en carton les petits spectroscopes c'est un réseau qu'y a / on a aussi utilisé un CD d'accord... [explication se référant à un modèle source lumineuse-prisme-écran projeté au tableau] on émet de la lumière on la fait passer par un truc qui la disperse alors évidemment ça sort en et moi j'ai mis un seul trait là pour le rayon de lumière mais ça sort vous le savez dispersé c'est là que ça vient le mot hein on disperse les couleurs OK »

Ensuite, s'appuyant sur un savoir déjà établi, l'enseignant marque une progression et introduit une nouvelle notion:

« et puis il y avait quelque chose qu'on avait complètement laissé de côté au début du chapitre trois certains avaient dit oui mais on voit aussi qu'il y a des petites rayures dans ces spectres c'est pas un problème d'imprimante hein y a vraiment des rayures fines et on avait fait l'hypothèse à ce moment-là que ça pouvait indiquer plein de choses sur la nature de l'étoile vous aviez fait toute une liste la taille la grosseur, l'activité le la composition chimique bon »

Dès la phase de préparation, l'enseignant annonce que c'est l'activité $5^{16}$ qui est à réaliser pendant la phase de travail en groupe. Il en rappelle l'énoncé et souligne le contenu à mobiliser, notamment la nécessité d'utiliser le modèle des spectres.

\section{Analyse locale}

Mais, comme dirait Sensevy (2007), comprendre l'action de l'enseignant c'est d'abord comprendre comment le contenu propre à cette action la spécifie, comment le contenu en spécifie en particulier l'évolution. Autrement dit, qu'en est-il ici des deux responsabilités didactiques de l'enseignant: d'une part, expliciter les modèles et les référents empiriques et d'autre part, initier l'élève à la dynamique référent empirique-modèle construit?

\section{Le référent empirique}

Le référent empirique comporte, par exemple, les supports "papier » représentant ${ }^{17}$ les modèles des spectres: 
(0:04:45.0) P « alors c'est pas facile de les reconnaître. On les collera tout à l'heure ne les collez pas tout de suite il y en aura peut-être en trop mais surtout ce qui vous paraît difficile »

Le référent empirique comporte aussi le phénomène étudié, par exemple la lumière d'une étoile

«parce que quand on analyse la lumière du soleil ou d'une étoile»

\section{Le modèle construit}

L'enseignant précise que:

« dans l'activité 5 à mon avis c'est que il faut prendre en compte à la fois le modèle sur les spectres d'émission et le modèle des spectres d'absorption»

\section{LApplication du modèle construit sur le référent empirique:}

L'enseignant rappelle le modèle établi la séance passée et fait référence à une représentation matérielle du modèle construit:

« regardez hein spectre du soleil simplifié il est là je vous le montre (P montre le spectre) vous l'avez déjà vu c'est le deuxième ça (montre) c'est le spectre de la lumière blanche parfaitement continu sans interruption sans raie noire voilà le spectre simplifié du soleil ( $\mathrm{P}$ le montre) c'est le même fond continu de la couleur blanche d'accord du rouge à gauche au bleu à droite même si souvent nous on présente l'inverse c'est pas grave ça veut juste dire qu'on a mis les grandes longueurs d'onde à gauche les petites longueurs d'onde à droite donc ne soyez pas perturbés par ça il y a pas de règle absolue là-dessus d'accord et vous voyez que dans les raies dans le spectre du émis par le soleil il y a des raies noires »

Dans l'énoncé de la consigne, il clarifie la tâche en termes de dynamique entre les deux registres empiriques et des modèles construits:

« parce que quand on analyse la lumière du soleil ou d'une étoile en général on va avoir à prendre en compte à la fois l'émission mais aussi les zones noires en particulier vous savez les traits noirs les raies noires dans le fond continu les raies noires c'est comme ça qu'on va le dire ces raies noires elles vont apparaître dans le spectre du soleil »

En outre, l'enseignant explique clairement que la représentation obtenue relativement au modèle d'absorption/émission dépend de la qualité du spectroscope utilisé.

« effectivement on peut difficilement faire mieux que voir ça (montre le spectre de lumière blanche) c'est-àdire que ils sont pas assez dispersifs ils dispersent pas la lumière assez précisément ces spectroscopes pour pouvoir voir les petites bandes noires (0:08:23.7) donc là il faut prendre un spectroscope beaucoup plus perfectionné d'accord le vieux spectroscope là très $\mathrm{XIX}^{\mathrm{e}}$ siècle que vous avez utilisé mardi à la limite celui-ci si on le pointait vers le ciel on pourrait commencer à voir les raies noires il faut déjà prendre un spectroscope de très bonne qualité »

L'enseignant conclut l'énoncé de la consigne en rappelant la tâche de modélisation en tant que dynamique entre modèle et référent empirique:

(0:06:28.3) P: «ce que vous demande le début de l'activité 5 et euh en utilisant le modèle et le document 2 c'est effectivement d'être capable d'interpréter pourquoi pour le soleil on a observation de ces raies noires »

Pendant la correction/structuration, l'enseignant reprend plutôt une série d'activités, de l'activité 1 à 3 , et un exercice proposé lors la séance antérieure (un TP) ${ }^{18}$. Il les reprend car il estime d'abord que ce rappel est nécessaire pour certains élèves, mais aussi parce qu'avec «le recul» que les élèves ont, ils peuvent résoudre les trois activités. Il les reprend également car il semble que le rappel des notions à mobiliser pour les corriger soit nécessaire pour la correction et l'explication de l'activité 5 . Ainsi, l'enseignant introduit la correction des activités en rappelant la définition du modèle «spectre d'émission »:

« on émet de la lumière on la fait passer par un truc qui la disperse alors évidemment ça sort et moi j'ai mis un seul trait là pour le rayon de lumière mais ça sort vous le savez dispersé c'est de là que ça vient le mot hein on disperse les couleurs OK donc ça sort dispersé et on met ça éventuellement sur un écran voilà et puis on fait une photo on l'a fait l'autre jour bien ça ça permet de 
dire ce que sont en général [ ] c'est le schéma général pour ce qui est spectre d'émission on émet de la lumière et on regarde de quoi elle est composée»

Il rappelle aussi la définition du modèle HR et en défini la signification et l'usage:

« les astrophysiciens travaillent sans arrêt avec ça les gens qui étudient les étoiles ils appellent ça le diagramme HR d'accord Hertzsprung-Russell c'est vraiment pour eux un moyen de d'échanger sans arrêt parce qu'ils disent tiens on a découvert une nouvelle étoile première chose qu'ils font quand ils publient la découverte ils disent elle est située à tel endroit dans le HR c'est ça c'est-à-dire que ça permet vraiment de cartographier les étoiles alors pas du tout les cartographier spatialement mais donner deux propriétés essentielles et ça va surtout dire des choses sur leur âge à quel stade de leur vie elles sont...»

Bref, l'analyse locale des actions de l'enseignant met en évidence que, que ce soit pour expliquer la consigne ou pour corriger les activités, ces actions traduisent bien les caractéristiques d'un processus de modélisation, notamment l'identification du réfé- rent empirique, la définition du modèle construit et l'application du second au premier en en précisant l'usage et les limites.

\section{Caractérisation locale des performances didactiques des élèves}

Que nous disent les performances didactiques cognitives sur les caractéristiques des constructions intellectuelles des élèves? Possèdent-elles les caractéristiques des constructions intellectuelles spécifiques aux processus de modélisation? Dans ce qui suit, des performances didactiques cognitives dans des moments clés de la séance sont explicitées, en vue d'identifier et de caractériser les constructions intellectuelles (modélisantes ou non) manifestées par les élèves. À titre d'illustration, sur la figure $\mathrm{n}^{\circ} 5$ sont repérés des moments où les élèves ont interagi avec l'enseignant, en posant des questions, en formulant des remarques ou des commentaires portant sur / en rapport avec le contenu en jeu. Par exemple, ces interactions ont porté sur: la signification d'un modèle, sa définition; le rapprochement entre un modèle et un savoir antérieur de l'élève qui

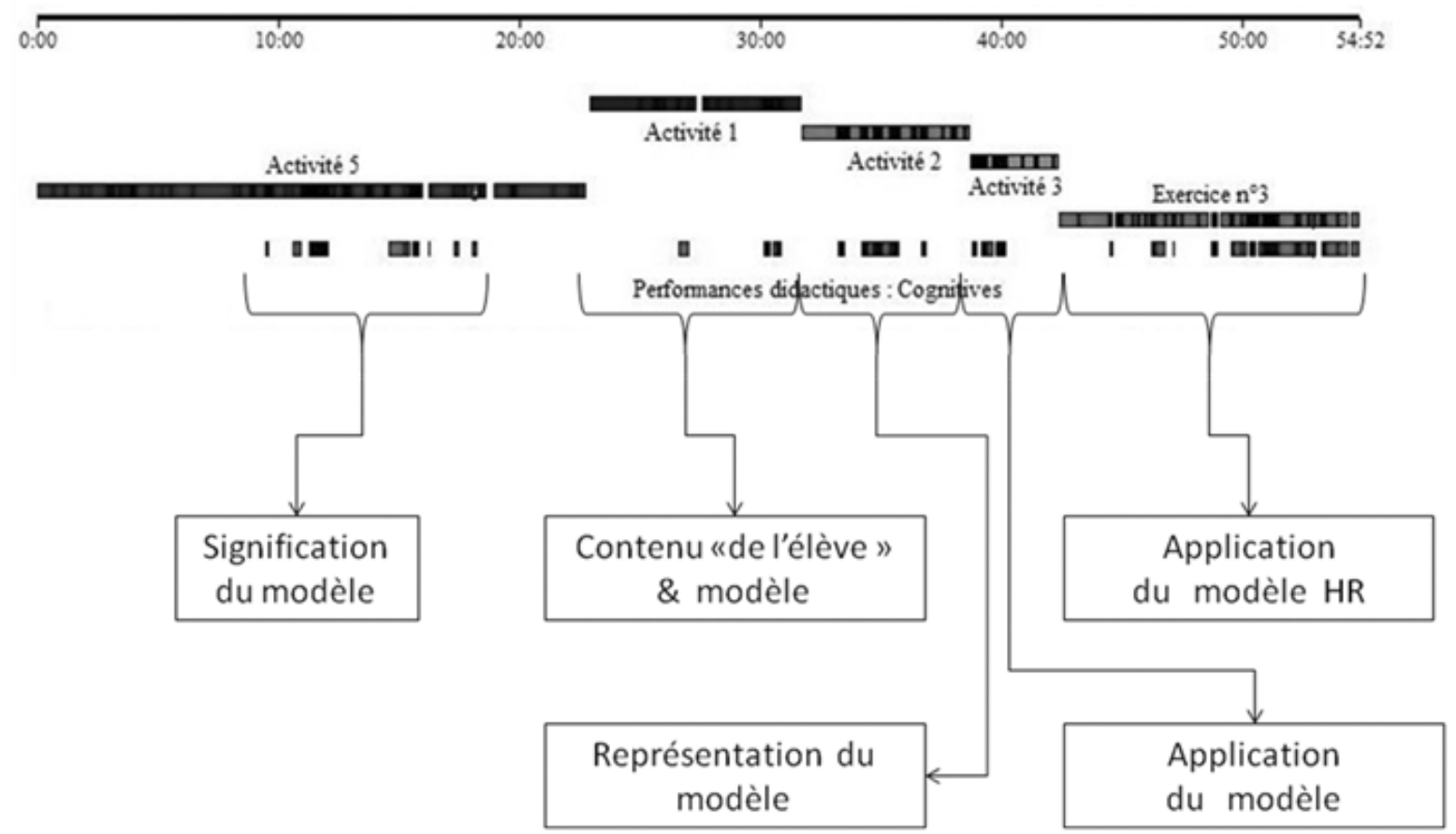

Figure $n^{\circ} 5$ : performances didactiques cognitives des élèves et modélisation 
peut relever de sa «culture générale» (luminosité des lucioles) ou d'une expérience vécue (persistance rétinienne) ${ }^{19}$; la représentation d'un modèle; l'application d'un modèle pour expliquer ou encore interpréter un phénomène.

\section{La signification d'un modèle}

L'élève pose une question sur la signification des modèles d'émission et d'absorption. L'enseignant en rappelle les définitions. L'élève discute ensuite de la différence des deux modèles et de quoi ils rendent compte.

E: monsieur il y a un truc qui me perturbe depuis beaucoup de séances

$\mathrm{P}$ : alors dis moi

E: c'est quoi la différence entre un spectre d'absorption et d'émission?

$\mathrm{P}$ : eh ben on va reprendre ça ensemble le spectre d'émission c'est un spectre qu'on obtient lorsque de la lumière est émise directement par une source d'accord? E:

$\mathrm{P}$ : et les spectres d'absorption regarde c'est le petit schéma qui est dans ton modèle ( $\mathrm{P}$ se déplace de son bureau vers l'élève qui est au premier rang) le spectre d'absorption c'est le spectre que tu obtiens si tu envoies de la lumière blanche qui traverse un objet transparent d'accord (montre sur la feuille du modèle)

$\mathrm{E}$ : et en fait comme la lumière elle est blanche là...

$\mathrm{P}$ : oui

E: si c'est pas passé par...

$\mathrm{P}$ : si y a pas de corps ici on obtient le spectre de la lumière blanche bien sûr

E: mais vous avez dit? directement? on obtient ça alors

$\mathrm{P}$ : ça c'est un spectre d'émission avec ce dispositif on a une source??? de la lumière blanche des vapeurs par exemple et on regarde le spectre

On voit bien que l'élève tente de comprendre le modèle, en particulier la reconnaissance et la distinction entre le spectre d'absorption et le spectre d'émission. Il est loin de se poser des questions sur le caractère hypothétique (c'est-à-dire voir comme si) de ce modèle, et encore moins de l'adapter et de percevoir sa pertinence pour d'autres situations.

\section{Le contenu «de l'élève»}

L'enseignant expliquait la différence entre la lumière émise par un néon et la lumière blanche émise par le soleil. Semblant vouloir donner un exemple dans le sens de ce que disait l'enseignant, l'élève fait référence au phénomène de la persistance rétinienne et à sa propre expérience de regarder «droit dans le soleil» et la perception qu'il en a après. Il se réfère donc à un contenu «empirique » et personnel. On peut penser que c'est une manière pour l'élève de chercher une explication au phénomène, en mobilisant les modèles expliqués par l'enseignant.

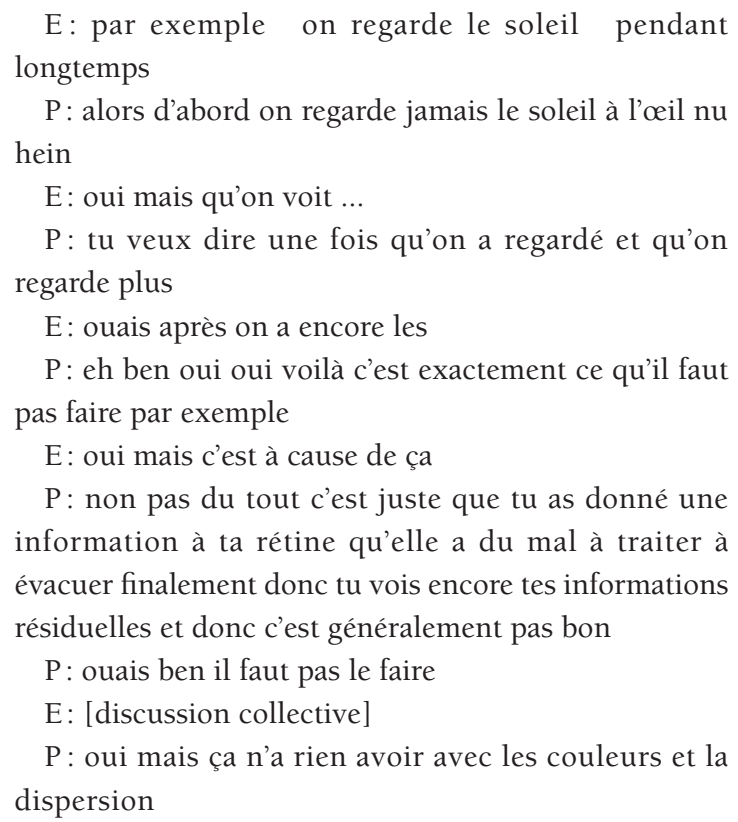

L'échange est rapide, et on peut même se demander si l'enseignant a compris le rapprochement que fait l'élève entre l'exemple qu'il donne et le modèle expliqué par l'enseignant. Mais ce n'est pas le seul moment de la séance où un élève se réfère à son «propre contenu » comme médiation par rapport au contenu proposé par l'enseignant. Un autre élève a posé une question sur l'émission de la lumière dans le cas des lucioles. C'est un autre exemple de contenu empirique et personnel amené par l'élève. Il fait le rapprochement avec ce que disait l'enseignant concernant le lien entre émission de la lumière, température de la source et type de spectre obtenu. 
P: c'est ce qui correspond maintenant au paragraphe Bl du modèle que je relis avec vous un solide ou un liquide émet de la lumière visible si sa température est suffisamment élevée alors je vous rappelle que nous à $37^{\circ}$ on émet du rayonnement des radiations monochromatiques mais dans l'infrarouge donc on nous voit pas briller dans la nuit, pas encore d'accord, parce qu'on émet on n'est pas assez chaud pour émettre dans le visible

E: Monsieur

$\mathrm{P}$ : oui

E: mais quand dans la nuit on voit des lucioles?

$\mathrm{P}$ : oui

E: c'est la lumière elle [est faite de la chaleur] ?

$\mathrm{P}$ : ah les lucioles c'est totalement autre chose c'est-àdire que les lucioles elles ont en elles quelque chose qui est capable d'émettre de la lumière dans le visible

E: et c'est quoi?

$\mathrm{P}$ : c'est totalement autre chose c'est une substance chimique qui est capable elle-même d'émettre sa lumière d'accord donc c'est vraiment autre chose

Il est intéressant de remarquer que parfois des élèves tentent ainsi de comprendre le modèle et son «fonctionnement» en tentant de faire le rapprochement avec des situations et des phénomènes expérimentés, vécus de manière plus personnelle.

\section{la représentation d'un modèle}

L'enseignant projette au tableau un spectre représentant les couleurs correspondant aux longueurs d'ondes dans le sens inverse de celui qu'ont les élèves sur leurs spectres. Les élèves se retrouvent en difficulté et sont conduits à poser des questions sur la possibilité de représenter le spectre dans les deux sens, la signification des graduations sur le spectre, la notion de longueur d'onde, etc.

E: si on l'a mis [le spectre longueurs d'ondes/ couleurs] dans l'autre sens

$\mathrm{P}$ : c'est pas grave non si vous l'avez mis dans l'autre sens comme vous voulez

$\mathrm{E}$ : en fait elles correspondent à quoi les graduations eh ben elles sont à l'inverse le bleu c'est le rouge c'est normal?

E: en fait elles correspondent à quoi les graduations

$\mathrm{P}$ : aux longueurs d'onde
E: ça aucun rapport avec la température les graduations

$\mathrm{P}$ : non, ben non la température c'est vers le bas que ça change

E: c'est pour ça (inaudible)

$\mathrm{P}$ : ben oui euh comment vous l'avez mis vous comme ça [P cherche sur son ordinateur] voilà bien, ça vous va mieux comme ça ( $\mathrm{P}$ a changé le sens du spectre et l'a projeté)?

$\mathrm{P}$ : non toujours pas vous voulez mettre le bleu à gauche vous c'est ça?

$\mathrm{E}$ : [parlent à plusieurs]

[P modifie le spectre puis le projette] $\mathrm{P}$ : ça vous va comme ça bien?

$\mathrm{P}$ : alors d'accord donc je répète ça c'était l'activité 2 il faut que vous soyez capable de faire le lien entre l'activité et le modèle (projette des photos et des spectres)

Sans doute, il semble qu'il y ait d'autres questions que les élèves souhaitent poser (l'enregistrement n'est pas clair pour le prouver), mais l'enseignant passe à l'activité suivante. L'essentiel des performances cognitives des élèves pendant ce moment a donc consisté à poser des questions pour tenter de stabiliser la signification et la représentation des modèles en jeu.

\section{L'application d'un modèle pour expliquer ou interpréter un phénomène}

À différents moments de la séance, le comportement des élèves laisse à penser qu'ils avaient pu s'approprier le modèle «spectre» et l'utiliser pour expliquer et classifier les températures de différentes étoiles.

$\mathrm{P}$ : ça y est c'est reparti qu'est-ce qui se passe alors qu'est-ce que vous en pensez là ( $P$ éteint la lumière du tableau) vous aviez à affecter à ces quatre températures quatre étoiles qui sont là c'était quoi les informations?

E: [réponses collectives inaudibles]

$\mathrm{P}$ : c'est l'influence de la température sur le spectre d'émission d'accord? En fonction de ce qu'on avait vu au chapitre

E: [bruit]

$\mathrm{P}$ chchuut alors qu'est ce que vous en pensez? P oui on s'écoute s'il vous plaît!

E: la première c'est bleuté parce qu'elle elle est chaude donc. 
$\mathrm{P}$ : voilà si la couleur est bleutée forcément l'étoile est très chaude la plus chaude donc ici c'était la température

E: $10000^{\circ}$

$\mathrm{P}$ : écrit au tableau dans le cadre $10000^{\circ}$

E: [à la surface?]

$\mathrm{P}$ : oui à la surface c'est marqué

On le constate, quelques réponses individuelles et collectives, énoncées avec l'aide de l'enseignant, laissent penser que certains élèves se sont appropriés la signification du modèle «spectre » et arrivent à l'adapter à l'activité de classification des étoiles en fonction de leurs températures. Un autre moment de la séance met en évidence le fait que les élèves s'approprient rapidement l'utilisation d'un autre modèle qui s'appuie sur l'analyse spectrale, le modèle HR. Ils arrivent à l'adapter à la situation proposée dans l' « exercice $\mathrm{n}^{\circ} 3$ ».

$\mathrm{P}$ : alors comment on les classe [quatre étoiles ayant des couleurs différentes] ben on regarde ça c'est une bleue en plus les noms sont mis en dessous hein c'est pour vous aider bleu blanc rouge et puis soleil [P montre au tableau les photos] à nouveau quatre étoiles voyez ? Alors on peut les repérer évidemment la plus froide il fallait les classer de la plus froide à la plus chaude la plus froide c'est la bleue

E: [réponse collective] c'est la rouge c'est la rouge... la blanche [la réaction de plusieurs élèves est très rapide]

$\mathrm{P}$ : euh la blanche excusez excusez-moi la rouge
Les élèves ont réagi rapidement et collectivement pour corriger l'erreur de l'enseignant lorsqu'il a considéré qu'«il fallait les classer [les étoiles] de la plus froide à la plus chaude, la plus froide c'est la bleue».

Ces constats nous permettent de dire que prises individuellement, ces performances mettent en évidence l'acquisition de la signification de certains modèles, et leur adaptation à des situations différentes. Elles ne permettent cependant pas d'apprécier par exemple si les élèves perçoivent le caractère hypothétique des modèles utilisés. Par ailleurs, la lecture transversale/temporelle de ces performances ne permet pas de dégager une cohérence qui signifierait une construction intellectuelle modélisante, caractérisée par la dynamique entre modèles et phénomène analysé, entre modèles et référents empiriques.

\section{Le silence: un moyen de contrôle ou une opportunité d'apprentissage?}

Nous rendons compte ici de l'évolution de l'action de l'enseignant, lorsqu'il agit sur les performances de silence, en étant guidé par deux questions. Comment l'enseignant a-t-il géré l'alternance entre silence et interactions collectives? Quel est le rôle des moments de silence qui semblent être si recherchés par l'enseignant dans cette séance? Les moments de silence analysés sont indiqués sur la figure $\mathrm{n}^{\circ} 6$.

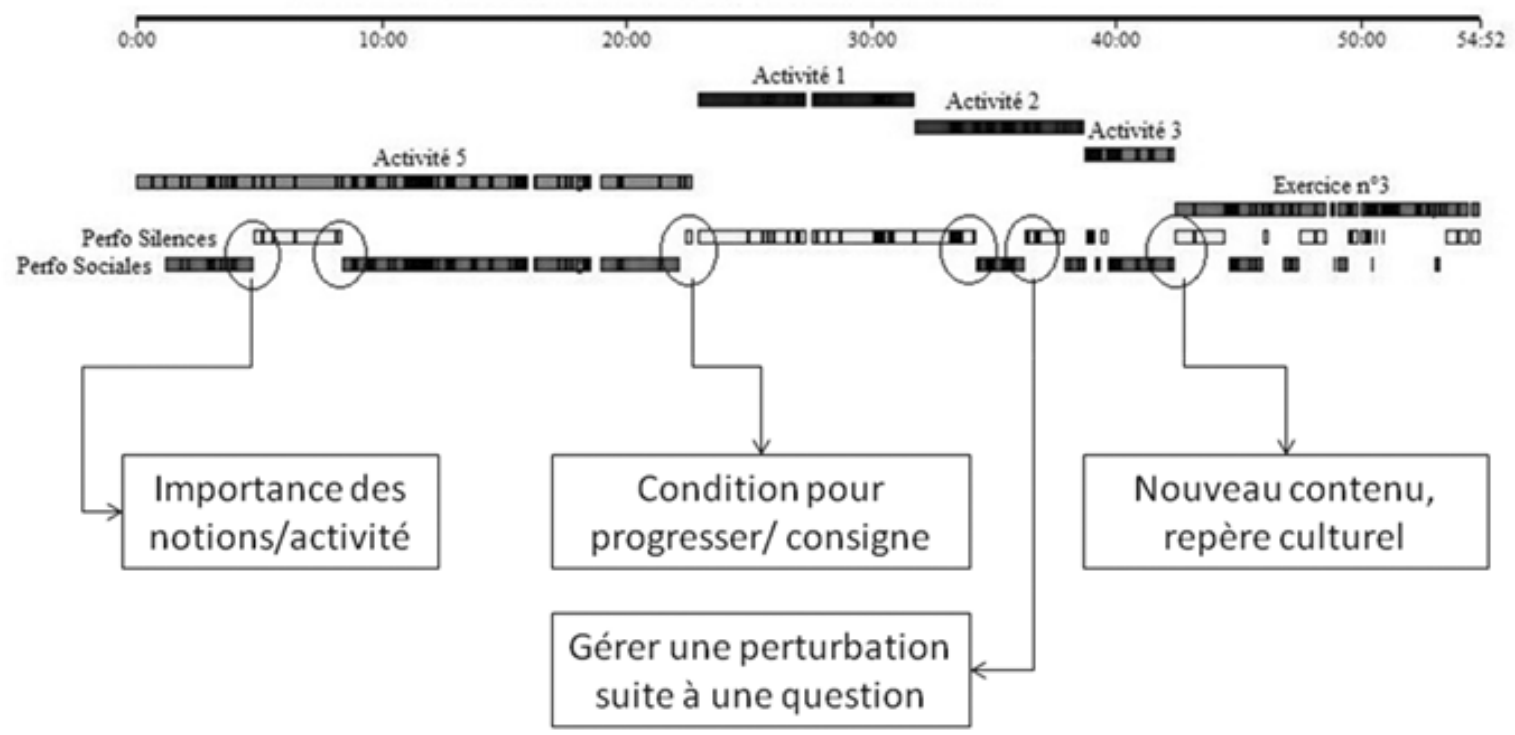

Figure $n^{\circ}$ 6: Alternances entre interactions et silences 
Dans le complexe de contraintes de la séance observée, la performance de silence semble être une variable d'ajustement décisive sur laquelle l'enseignant agit pour progresser. Mais c'est une variable d'ajustement qui a eu différents statuts et fonctions au cours de la séance: une conséquence d'un intérêt particulier des élèves à un contenu par rapport à sa nouveauté, son utilité ou sa valeur culturelle; une condition pour l'enseignant pour progresser dans la séance; une routine (Lacourse, 2008) pour gérer une perturbation, etc.

\section{Un silence suscité par l'intérêt des élèves à une notion nécessaire à la réalisation de l'activité}

Au début de l'énoncé de l'activité 5, les élèves chuchotent plus ou moins bruyamment. Ils prêtent leur attention progressivement, et le silence commence à s'installer quand l'enseignant évoque le contenu nécessaire pour la réalisation de cette activité (le modèle des spectres, la différence entre spectre d'émission et spectre d'absorption):

$\mathrm{P}$ : [continue à chercher dans ses documents Il prend quelques documents et se dirige vers les élèves] alors vous commencez puisque vous avez fait ça chez vous on repasse au mode activité vous discutez avec votre voisin ou votre voisine pour confronter vos réponses à deux ça sera peut-être plus facile en échangeant que tout seul attention vous avez pour faire ce travail-là le droit voire le devoir d'utiliser le modèle des spectres et en particulier puisqu'on en est là vous avez été lire on l'a lu petit à petit ce modèle vous avez maintenant à votre disposition le paragraphe c qui s'appelle spectre d'absorption

E: c'est pas facile de les reconnaître

P : alors c'est pas facile de les reconnaître. On les collera tout à l'heure ne les collez pas tout de suite il y en aura peut-être en trop mais surtout ce qui vous paraît difficile dans l'activité 5 à mon avis c'est que il faut prendre en compte à la fois le modèle sur les spectres d'émission et le modèle des spectres d'absorption

Ce silence est vraisemblablement lié à l'importance des notions de spectre d'absorption et spectre d'émission. L'analyse précédente montre bien que les difficultés des élèves se situent à ce niveaulà et que ces notions sont la clé de voûte pour comprendre et effectuer les activités proposées. Le silence est rompu lorsque le rappel de cours prend fin, et précisément, dès que l'enseignant raccroche le poster des spectres sur le mur et invite les élèves à réfléchir en groupe

Un silence suscité par l'intérêt des élèves à une notion nouvelle, ayant une valeur culturelle

Pour rétablir le silence, l'enseignant a procédé par moments sans recours à la routine de «rappel à l'ordre». Ce qui est privilégié dans l'extrait suivant c'est l'introduction d'un nouveau contenu (le modèle HR), en expliquant son utilisation et, dans une sorte de «repère culturel », en le référant à la pratique sociale des astrophysiciens.

$\mathrm{P}$ : oui [se penche sur son ordinateur]

E: [discussions, bruit]

$\mathrm{P}$ : voilà

$\mathrm{P}$ : [P retrouve la page à projeter] c'était cet exercice ben regardez on va vérifier un petit peu

P: [efface le tableau]

$\mathrm{P}$ : quand on a fait l'activité ça vous a pas fait penser à cet exo, c'est exactement la même chose, sauf qu'on en sait un peu plus sur le spectre d'émission. Alors regardez, [P a projeté au tableau l'exercice] bon alors c'est bon? C'est un peu volontaire de ma part c'est un exo que j'ai pris dans un manuel scolaire hein

$\mathrm{P}$ : mais c'est un diagramme à droite là [P montre sur le tableau]. Bon, les astrophysiciens travaillent sans arrêt avec ça. Les gens qui étudient les étoiles ils appellent ça le diagramme HR d'accord, Hertzsprung-Russell, c'est vraiment pour eux un moyen d'échanger sans arrêt

\section{Un silence/condition pour progresser d'une phase dans la séance à une autre}

Pour ramener les élèves au silence au début de la phase de structuration/correction, l'enseignant rappelle à l'ordre un élève au fond de la classe. Il prend son temps pour le faire et en profite pour annoncer son intention de faire un rappel des notions nécessaires pour réaliser l'activité 5 :

«P : alors on reprend ensemble s'il vous plaît

$\mathrm{P}$ : Paul, il me semble que tu as des trucs à rattraper que tu as rien compris parce que tu étais pas là mais en discutant à trois mètres de là; je suis pas sur que ça 
t'aide beaucoup, alors moi je veux bien t'aider, faire un petit rattrapage rapide, mais c'est maintenant que ça se passe.

E [commentaire de l'élève] Je vous écoute

$\mathrm{P}$ : oui mais plus que ça tu aurais pu collaborer un peu avec tes voisins comme je te l'avais demandé, bon.»

Ce temps lui permet de réinstaller le silence en classe et de commencer la correction. Cette situation d'exposition de l'enseignant et de silence du côté des élèves permet à ceux-ci d'intervenir sporadiquement (quatre fois) pour émettre des questions ou des commentaires, que nous considérons donc comme des performances cognitives.

\section{Un silence/condition pour progresser dans l'explication d'une notion}

Le silence des élèves n'a pas constitué uniquement une condition pour progresser d'une phase de la séance vers une autre. Mais il a constitué aussi une condition pour avancer dans l'explication d'une notion, comme le montre cet extrait.

$\mathrm{P}$ : on émet de la lumière on la fait passer par un truc qui la disperse alors évidemment ça sort et moi j'ai mis un seul trait là pour le rayon de lumière mais ça sort vous le savez dispersé c'est là que ça vient le mot hein on disperse les couleurs OK donc ça sort dispersé et on met ça éventuellement sur un écran voilà et puis on fait une photo on l'a fait l'autre jour bien ça ça permet de dire ce que sont en général

$\mathrm{P}$ : [à deux élèves qui n'écoutent pas derrière] vous me direz hein quand je peux y aller. Si je vous dérange vous me prévenez.

$\mathrm{P}$ : c'est le schéma général pour ce qui est spectre d'émission, on émet de la lumière et on regarde de quoi elle est composée. »

\section{Un silence/routine pour confronter une perturbation imprévue}

Une question «imprévue» d'un élève par rapport à la différence entre le spectre projeté au tableau et celui que les élèves ont, différence en ce qui concerne l'ordre des couleurs représentant les longueurs d'ondes, précipite les élèves dans des interactions diffuses, désordonnées, entre eux ou avec l'ensei- gnant. L'enseignant essaye de reprendre l'explication en utilisant le vidéoprojecteur, mais il est finalement obligé de recourir à un rappel à l'ordre de « certains » élèves sans les nommer, en s'adressant à la classe entière:

«P : alors d'accord donc je répète, ça c'était l'activité 2 , il faut que vous soyez capable de faire le lien entre l'activité et le modèle (projection des photos et des spectres)

\section{E: monsieur}

P : un métal chauffé très fort ou un corps chauffé très très fort à très haute température je sens qu'il y en a qui vont pas finir l'heure. Je le sens comme ça. [le silence revient] Ça va émettre un spectre non seulement continu mais dont l'allure la répartition des couleurs va dire une idée, va donner une idée de la température

À partir de cet instant (au début de l'activité 2), les moments de silence sont de plus en plus courts et alternent avec des moments d'interaction désordonnés et bruyants de plus en plus longs, mais pendant lesquels l'enseignant arrive quand même à traiter les activités 2 et 3 ainsi que l'exercice 3 , non sans difficultés. Ainsi, la manifestation des performances de silence par les élèves ou la recherche de ces performances par l'enseignant est déterminée par, entre autres, l'énoncé d'une consigne, un rappel et/ ou une explication d'un contenu à mobiliser, l'introduction d'un nouveau contenu en lien avec une référence culturelle, une explication utilisant un support (tableau, document, matériel), bref, une prise de décision didactique et pédagogique qui mobilise l'intérêt des élèves. La manifestation des performances de silence tient aussi aux rappels à l'ordre, un moyen auquel l'enseignant a souvent recours pour se recentrer rapidement sur les autres contraintes de la situation. Par ailleurs, l'interruption du silence tient par exemple à l'incompatibilité des instruments de travail (modèles de spectre sur papier) entre élèves et enseignant, à un contenu non préparé par l'enseignant et révélé par une question inattendue (par exemple la question sur l'ordre des couleurs du spectre), à un savoir mal formulé par l'enseignant, à un manque de ressource (document, support) ou à une réponse attendue par les élèves.

Cette description ne nous permet pas d'affirmer la construction par les élèves de processus intellectuels modélisants pendant les moments de silence. 
Pour caractériser le silence et son rôle dans l'action d'enseignement/apprentissage, on ne peut pas faire l'économie d'une étude plus approfondie, qui donne la parole aux élèves et à l'enseignant, pour sonder ce qui se passe pendant les moments de silence (par exemple, à travers l'analyse des interactions qui succèdent à un silence ou l'analyse des moments d'écoute d'une explication, etc.).

\section{Conclusion}

L'exercice qui nous est proposé dans ce numéro thématique est difficile. Il s'agit de se saisir d'un objet de recherche à partir de l'enregistrement vidéo réalisé d'une situation d'enseignement/apprentissage. $\mathrm{Au}$ regard des choix ${ }^{20}$ faits par le chercheur qui a filmé (le réalisateur), notre point de départ est en fait une première interprétation de la situation. Notre intérêt en tant que chercheur doit alors composer d'une part, avec le respect de la commande (une donnée importante du problème) et d'autre part, avec la pertinence de l'approche adoptée par rapport au corpus «donné », mais aussi à reconstruire. Deux caractéristiques essentielles de la technologie vidéo permettent ce travail de reconstruction et de réappropriation de la situation à analyser. D'une part, la permanence (Pirie, 1996; Engle, Conant et Greeno, 2007), en ce sens que l'enregistrement vidéo conserve aussi bien les éléments essentiels de la situation que les traces des choix faits par le réalisateur (caméra au fond de la classe, grand angle sur les élèves et le professeur, des mouvements de zoom pour mettre en évidence des événements particuliers de la situation, etc.). Ces traces facilitent en effet l'entrée dans ce que Lemke (2007) appelle les attentional worlds, de la séance filmée mais aussi ceux du réalisateur et ses présupposés. D'autre part, la possibilité d'un travail collaboratif (DuFon, 2002; Erickson, 2006) entre chercheurs pour «contrôler» leurs interprétations (en ce qui nous concerne ici l'interprétation de départ ${ }^{21}$ ) et pour optimiser la validité des résultats.

L'enregistrement vidéo en tant que tel présente une séance d'enseignement en sciences où un complexe de contraintes pèse sur l'action de l'enseignant et sur son évolution. Notre analyse vise à dénaturaliser ce que la vidéo donne à voir de prime abord, en particulier l'omniprésence de l'enseignant, la passivité de l'élève et une interaction unidirection- nelle de l'enseignant vers l'élève. L'analyse de l'action de l'enseignant, conçue comme double médiation, une action sur les performances didactiques des élèves, permet en effet de dévoiler une évolution de l'action plus subtile. L'évolution de l'action de l'enseignant est déterminée ${ }^{22}$ par des prises de décisions par l'enseignant pour faire évoluer la séance à travers ses différentes phases. En même temps, à l'intérieur de chaque phase, les performances didactiques des élèves constituent un moteur essentiel, et non exclusif, de l'évolution du système didactique. Que ce soit pour expliquer la consigne ou pour corriger les exercices, l'action de l'enseignant manifeste de manière constante les caractéristiques d'un processus de modélisation, c'est-à-dire une dynamique entre référent empirique et modèle construit. Par ailleurs, les performances manifestées par les élèves mettent en évidence l'appropriation de la signification de certains modèles et la capacité de les adapter à des situations différentes. Cependant, elles ne permettent pas de conclure quant à une construction intellectuelle à caractère modélisant.

Mais au-delà de ces quelques résultats, un des objectifs de cette contribution est aussi de présenter une première tentative d'opérationnalisation de la notion de performance didactique. Le sens donné à la notion de performance se limite souvent à mettre en correspondance, selon un processus implicite, performance et apprentissage, performance et acquisition des savoirs ou encore performance et acquisition conceptuelle (Tiberghien, Malkoun, Buty, Souassy et Mortimer, 2007). La performance didactique est mobilisée ici en tant que construit qui vise à donner à voir la dynamique de l'évolution de l'action d'enseignement (Tiberghien et Sensevy, 2011). C'est un construit qui vise à rendre compte de l'acquisition d'un contenu et du processus social nécessaire pour l'acquérir, d'où la distinction heuristique entre performances sociales, cognitives, techniques et de silence. Considérer le silence de l'élève comme performance vise, encore une fois, à le dénaturaliser comme phénomène très recherché par l'enseignant, du moins dans la séance analysée, et vécu par les élèves mais souvent ignoré et non pensé. Nous pensons que la connaissance du silence ${ }^{23}$ est fondamentale pour comprendre les processus d'enseignement et d'apprentissage; cette position n'évacue pas le statut et le rôle ambigus voire énigmatiques du silence dans la pratique d'enseignement et d'appren- 
tissage. Par exemple, comment juger de la valeur didactique (Reuter, 2005) d'un silence? Comment différencier les silences dus à la réflexion, à la timidité, à la résistance ou à l'évitement? Etc.

Cette analyse ne fait donc qu'effleurer la question de l'interdépendance des performances didactiques et l'action de l'enseignant. Dans une perspective de recherche ou d'intervention, l'étude pourrait se prolonger par exemple par l'analyse des performances didactiques des élèves que des situations d'enseignement/apprentissage en sciences permettraient de favoriser. Elle pourrait aussi se prolonger par l'analyse des performances didactiques recherchées, valorisées par des acteurs éducatifs, et identifier d'une part, les actions de l'enseignant qui permettraient de les entretenir et de les renforcer dans une situation didactique et, d'autre part, les environnements qui favoriseraient un type particulier d'évolution du système didactique (Tiberghien et al. 2007). 


\section{NOTES}

1. Entretien réalisé par Laurent Talbot, dans le cadre de ce travail d'analyse croisée d'une même unité d'enregistrement.

2. Ces contraintes se situent à plusieurs niveaux. Au niveau temporel, en ce sens que la séance est programmée trois jours après la séance en groupe où les notions de bases ont été introduites et que le contenu traité par l'enseignant est très dense au regard de la durée de la séance observée (54mn); au niveau spatial, en ce sens que la salle est bruyante et ne permet pas à l'enseignant de circuler entre ses élèves; au niveau interactionnel, en ce sens que l'enseignant, avec une classe entière de 36 élèves dont « quatre à cinq élèves sont difficiles à canaliser $»$, fait travailler en groupe. Il introduit de nouveaux contenus, à travers une suite d'activités d'application, dans le cadre d'un programme ayant une configuration thématique.

3. La disqualification rapide de telles situations d'enseignement empêche de comprendre, par exemple, les ressorts des résultats positifs des pratiques d'enseignements dans des pays où les approches dites « transmissives » ou «frontales » sont des pratiques courantes, en particulier le Japon et Hong Kong (De Marcellus, 2011).

4. Cela ne suppose pas une évolution temporelle linéaire de l'action de l'enseignant.

5. Pour plus de développements, voir Yves Lenoir (2009), document accessible en ligne au http://ncre.educ. usherbrooke.ca/articles/v12n1/01_NCre12_1Len.pdf.

6. Séminaire de recherche de l'équipe Théodile-CIREL (2007-2009): Performances et apprentissages disciplinaires. Les contributions à ce séminaire ont fait l'objet de deux numéros de la revue Les Cahiers Théodile (actuellement Recherches en didactiques - Cahiers Théodile) numéros 8 et 9.

7. PISA: Programme for International Student Assessment; TIMSS: Trends in International Mathematics and Science Study.

8. La notion de silence ainsi que la limite des liens supposés d'une part entre performance et réussite et d'autre part entre non performance et échec ont été discutées par Jovenet (2009) en mobilisant les deux concepts de « performance désir » et « performance évitement ». Malgré l'intérêt de cette approche psychanalytique pour la didactique, sa pertinence pour notre propos est conditionnée par la mise en relation du désir ou de l'évitement avec l'apprentissage d'un contenu spécifique (disciplinaire ou non), ce qui reste à établir.

9. Nous reprenons ici le terme utilisé par l'enseignant: applications renvoie à exercices permettant de mettre en application des notions en physiques vues dans la séance précédente.

10. En fait, il y avait deux caméras dans la salle dont une pour suivre l'enseignant, mais la caméra élève n'a pas permis de capter le son suite à un problème technique.

11. Un diagramme dit de Hertzsprung-Russell utilisé par les astrophysiciens pour situer les étoiles dans l'espace.

12. Transana: un logiciel d'aide à l'analyse d'enregistrements vidéo, http://www.transana.org/

13. L'élève dans ce cas manifeste une attitude d'attention à ce qui est dit ou fait. 
14. Les interactions entre les élèves ou avec l'enseignant ne portant pas directement sur un contenu. Elles portent plutôt sur les conditions de la mobilisation du contenu, de sa présentation; sur sa construction (objectifs de l'activité, documents ressources) et sur ses caractéristiques (difficile, facile, etc.).

15. Pour chaque enseignante, le premier événement qui correspond au début de l'enregistrement vidéo n'est pas pris en compte, et donc le nombre d'événements considérés est égal au nombre de segments identifiés.

16. L'enseignant reprend la correction de l'activité 5 après la pause (ce que ne représente pas ce graphe).

17. Remarquer ici comment une représentation matérielle du modèle construit la séance précédente se transforme en un élément du référent empirique. Pour dire que la proposition de Martinand (2010) de considérer la modélisation comme une dynamique entre référent empirique et modèles construits est plus précise que de la concevoir comme des allers et retours entre « le réel » et le symbolique.

18. Notre analyse a consisté ici à choisir les moments marquants dans la structuration, en ce qui concerne les trois activités et l'exercice d'application. Nous avons systématiquement précisé ce que fait l'enseignant pour expliquer le processus de modélisation en se basant sur des applications différentes.

19. Nous avons appelé ces ressources amenées par les élèves en classe 'contenu « de l'élève »' sur la figure n 5 .

20. Notamment sa question de recherche: comment l'enseignant établit le contrat didactique avec ses élèves dans une situation d'enseignement/apprentissage en sciences?

21. En ce sens, l'enregistrement vidéo réalisé par Laurent Talbot nous a été particulièrement utile.

22. Ce résultat reflète l'interprétation du chercheur et ne tient pas compte de l'interprétation de l'enseignant, ce qui est une limite de notre analyse. La prise en compte de l'interprétation de l'enseignant permettrait-elle de corriger/préciser le nombre d'événements liés à des prises de décisions de l'enseignant (75 événements) ? C'est possible, à croire la recherche de Morine et Valance (1976) citée par Crahay (1989), le nombre moyen de décisions prises de façon délibérée (rationnelle?) pendant une leçon se situe entre 9,6 et 13,9.

23. En tant que faire qui a une présence "pratique » et existentielle en classe, le silence peut être considéré comme un événement performance, un événement performatif. Le silence n'est ni du mutisme ni une « absence de son », il comporte une activité consciente (Dauenhauer, 1980), c'est un langage implicite (Merlau-Ponty, 1979).

\section{RÉFÉRENCES}

Astolfi, J.- P. (1992). L'école pour apprendre. Paris: ESF (Collection Pédagogiques).

Bautier, E. (2006). Le rôle des pratiques des maîtres dans les difficultés scolaires des élèves. Une analyse de pratiques intégrant la dimension des difficultés socialement différenciées. Recherche et Formation, 51, p. 105-118.

Chopin, M-P. (2008). La visibilité didactique: un milieu pour l'action du professeur. Présentation d'un concept pour l'étude des pratiques d'enseignement. Éducation et didactique, 2, 2, p. 63-79

Crahay, M. (1989). Contraintes de situation et interaction maître-élève. Changer sa façon d'enseigner, est-ce possible? Revue française de pédagogie, 88, p. 77-94. 
Dauenhauer, B. P. (1980). Silence: The phenomenon and its ontological significance. Bloomington.

Daunay B. (2008). Performances et apprentissages disciplinaires. Cahiers Théodile $\mathrm{n}^{\circ}$ 8, Villeneuve d'Ascq, Université Charles de Gaulle - Lille 3, p. 7-23.

Daunay, B. (2007). Système didactique - triangle didactique. In Y. Reuter (2007) (dir.), Reuter, Y. (2007). Dictionnaire des concepts fondamentaux des didactiques (p. 209-216). Bruxelles: De Boeck Université.

De Marcellus, O. (2011). Courants et tourbillons. Gestions dévolutives et directives en classe. Actes du Symposium International Recherches sur l'utilisation de la vidéo dans la formation d'enseignants de mathématiques et de sciences (p. 49-51), CADIVAM, Lausanne, 23-25 juin 2011, Haute École Pédagogique, Lausanne, Suisse.

Dufon, M. A. (2002). Video recording in ethnographic SLA research: some issues of validity in data collection. Language Learning \& Technology, 6, 1, p. 40-59.

Engle, R. A., Conant, F. C. et Greeno, J. G. (2007). Progressive refinement of hypotheses in video-supported research. In R. Goldman, R. Pea, B. Barron et S. Derry (dirs.), Video research in the learning sciences. Mahwah, NJ: Erlbaum.

Erickson, F. (2006). Definition and analysis of data from videotape: Some research procedures and their rationales. In J. Green, G. Camilli \& P. Elmore (dirs.), Handbook of Complementary Methods in Éducation Research. Mahwah, NJ: Erlbaum.

Freitag, M. (1986). Dialectique et société. Tome I: Introduction à une théorie générale du Symbolique. Montréal: Éditions coopératives Albert Saint-Martin.

Johsua, S. et Dupin, J-J. (1993). Initiation à la didactique des sciences et des mathématiques. Paris: PUF.

Jovenet, A-M. (2009). Rencontre improbable entre deux concepts: silence et performance. Les Cahiers Théodile, 9, p. 101-122.

Lacourse France (2008) «Les routines professionnelles en formation initiale. De la prescription à l'analyse des pratiques », Recherche et formation, $n^{\circ} 59$, p. 141-154.

Lahanier-Reuter D., (2008). Performances et apprentissages en didactique des mathématiques. Les Cahiers THEODILE nº 9 , p. 45-60.

Lemke, J. (2007). Video Epistemology In-and-Outside the Box: Traversing Attentional Spaces. In R. Goldman, R. Pea, B. Barron et S. Derry (dirs.), Video research in the learning sciences. Mahwah, NJ: Erlbaum.

Lenoir, Y. (1996). Médiation cognitive et médiation didactique. In C. Raisky et M. Caillot (dir.), Le didactique au-delà des didactiques. Débats autour de concepts fédérateurs (p. 223-251). Bruxelles: De Boeck Université.

Lenoir, Y. (2009). L'intervention éducative, un construit théorique pour analyser les pratiques d'enseignement. Les nouveaux cahiers de la recherche en éducation, 12(1), p. 9-29.

Martinand, J.-L. (2010). Schémas didactiques pour la modélisation en sciences et technologies. Spectre, 40, 1, p. $20-24$. 
Maubant, P. et Zaid, A. (à paraître). L'analyse des pratiques d'enseignement: éléments de cadrage et rationnel sous-tendant le dispositif méthodologique. In J. Clanet (dir.), Regard pluriel sur le travail enseignant. Paris: L'Harmattan.

Merlau-Ponty, M. (1979). Le visible et l'invisible. Paris, Éditions Gallimard.

Orange, C. (1997). Problèmes et modélisation en biologie: quels apprentissages pour le lycée? Paris: PUF.

Pirie, S. E. B. (1996). Classroom video-recording: when, why and how does it offer a valuable data source for qualitative research? Paper presented at the $18^{\text {th }}$ annual meeting of the North American chapter of the international group for the psychology of mathematics education, octobre 1996, Panama City, Florida. ERIC ED 401 128, 17 pages.

Reuter Yves (dir.) (2005), Pédagogie du projet et didactique du français, Penser et débattre avec Francis Ruellan, Villeneuve d'Ascq, Presses Universitaires du Septentrion.

Reuter, Y. (2007) (dir.). La conscience disciplinaire: présentation d'un concept. Éducation et didactique, 1, 2, p. 57-72.

Reuter, Y. (2011). À propos de la notion de performance en didactiques : Éléments de discussion. Recherches en didactiques - Les Cahiers Théodile, 11, p. 129 - 142.

Sarrazy B. (2008). Différencier les hétérogénéités dans l'enseignement des mathématiques: tenants idéologiques et enjeux didactiques. In A. Rouchier et I. Bloch, Actes de la XIII école d'été de Didactique des Mathématiques (Sainte-Livrade, Lot-et-Garonne, du 18 au 26 août 2005). Grenoble: la Pensée Sauvage.

Sensevy, G. (2007). Des catégories pour décrire et comprendre l'action didactique. In G. Sensevy et A. Mercier (dirs.), Agir ensemble. Agir ensemble. L'action didactique conjointe du professeur et des élèves (p. 13-49). Rennes: Presses Universitaires de Rennes.

Tiberghien, A., Malkoun, L., Buty, C., Souassy, N. et Mortimer, E. (2007). Analyse des savoirs en jeu en classe de physique à différentes échelles de temps. In G. Sensevy et A. Mercier (dirs.), L'action didactique conjointe du professeur et des élèves (p. 13-49). Rennes: Presses Universitaires de Rennes.

Tiberghien, G. et Sensevy, G. (2011). À propos des relations dynamiques entre les déterminants de l'action didactique conjointe et les performances des élèves. Communication au $2^{e}$ colloque international de l'ARCD: Les contenus disciplinaires, Villeneuve d'Ascq, 20-22 janvier 2011.

Zaid, A. et Cohen-Azria, C. (2011). La pratique d'enseignement conçue comme action sur les performances didactiques des élèves. Exemple d'analyse d'enregistrements vidéo de deux séances en sciences à l'école primaire. Actes du Symposium International Recherches sur l'utilisation de la vidéo dans la formation d'enseignants de mathématiques et de sciences (p. 46-48), CADIVAM, Lausanne, 23-25 juin 2011, Haute École Pédagogique, Lausanne, Suisse. 